\title{
Acknowledgment to Reviewers of Pharmaceutics in 2020
}

\section{Pharmaceutics Editorial Office}

MDPI AG, St. Alban-Anlage 66, 4052 Basel, Switzerland

Citation: Pharmaceutics Editorial Office. Acknowledgment to Reviewers of Pharmaceutics in 2020 Pharmaceutics 2021, 13, 154 https://doi.org/10.3390/ pharmaceutics13020154

Published: 25 January 2021

Publisher's Note: MDPI stays neutral with regard to jurisdictional claims in published maps and institutional affiliations.

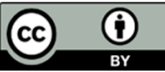

Copyright: () 2021 by the author. Licensee MDPI, Basel, Switzerland. This article is an open access article distributed under the terms and conditions of the Creative Commons Attribution (CC BY) license (http://creativecommons.org/licenses/by/4.0/).

Peer review is the driving force of journal development, and reviewers are gatekeepers who ensure that Pharmaceutics maintains its standards for the high quality of its published papers. Thanks to the cooperation of our reviewers, in 2020, the median time to first decision was 14 days and the median time to publication was 35 days. The editors would like to express their sincere gratitude to the following reviewers for their precious time and dedication, regardless of whether the papers were finally published:

Abakumov, Maxim A.

Abatzoglou, Nicolas

Abbina, Srinivas

Abd El Hakim, Yasmina

Abdel-Daim, Mohamed

Abdeltawab, Nourtan

Abejón, Ricardo

Abraham, Wolf-Rainer

Abruzzo, Angela

Acedo Nunez, Maria Del Pilar

Acharya, Ghanashyam

Acri, Giuseppe

Adebisi, Adeola O.

Aderibigbe, Blessing

Aderibigbe, Blessing Atim A.

Adil, Syed Farooq

Adobes-Vidal, Maria

Adorni, Maria Pia

Adrover, Alessandra

Adunyah, Samuel E.

Afshari, Khashayar

Afzal, Adeel

Agatemor, Christian

Agrahari, Vivek

Aguirre, Aitor

Aguirre-Álvarez, Gabriel

Ahad, Abdul

Ahmad, Ali

Ahmad, Javed

Ahmad, Zeeshan

Ahmed, Marya

Ahmed, Osama A. A.

Aigner, Zoltán

Airaksinen, Anu J.

Aitipamula, Srinivasulu

Akagi, Yuki
Akhlaghi, Seyedeh Parinaz

Akita, Sadanori

Al Qahtani, Mohammed

Alanis-Lobato, Gregorio

Alavi, Seyed Ebrahim

Albaladejo Vicente, Romana

Albericio, Fernando

Albrechtsen, Nicolai J. Wewer

Alcudia, Ana

Aldred, Katie

Aleandri, Simone

Alegret, Nuria

Alessandro, Cecconello

Alexander, Cameron

Alexa-Stratulat, Teodora

Al-Fatimi, Mohamed

Alfei, Silvana

Alghamdi, Hamdan S.

Al-Gousous, Jozef

Alhakamy, Nabil

Alhareth, Khair

Ali, Adel A

Aljayyoussi, Ghaith

Alkilani, Ahlam Zaid

Allegaert, Karel

Allen, Sean David

Almoazen, Hassan

Almoazen, Hassen

Alonso-Moreno, Carlos

Alsaab, Hashem

Al-Tabakha, Moawia M.

Altmann, Friedrich

Altucci, Lucia

Alves, Carlos

Alves, Francisco

Ambrus, Rita 
An, $\mathrm{Yu}$

Ananthakrishnan, Soundaram

Jeevarathinam

Ando, Koji

Andrić, Filip

Andriolo, Jessica

Angela Barba, Anna

Angelopoulou, Athina

Angelov, Borislav

Angelova, Angelina

Angelucci, Emanuele

Angsantikul, Pavimol

Anne-Claire, Groo

Anraku, Makoto

Ansari, D. Mohd. Azam

Ansari, Mohammad Javed

Ansorena, Eduardo

Antal, István

Antimisiaris, Sophia G.

Antipina, Maria N.

Antoniac, Iulian

Antoniu, Sabina Antonela

Anzenbacherova, Eva

Aparicio-Blanco, Juan

Apostolopoulos, Vasso

Aquavella, James V.

Aran, Veronica

Arana, Lide

Araújo, Thaíse Gonçalves

Ariatti, Mario

Ariga, Katsuhiko

Armakovic, Sanja J.

Armanini, Decio

Arnolds, Heike

Arufe, María C

Arunachalam, Prabhakarn

Ashihara, Eishi

Ashley, Jon

Aso, Yoshimasa

Augustyniak, Adrian

Aungst, Bruce

Avgoustakis, Konstantinos

Awasthi, Vibhudutta

Azam, Faizul

Azer, Samy A.

Azevedo, Nuno F.

Azzimonti, Barbara

Babič, Michal

Babincová, Melánia

Baby, André Rolim

Bacci, Stefano
Bach, Horacio

Bácskay, Ildikó

Badea, Ildiko

Badea, Nicoleta

Badimon, Juan J.

Bado, Igor

Badr, Gamal

Bae, Jeehyeon

Baek, In-Hwan

Baer, Patrick C.

Bagyinszky, Eva

Bahal, Raman

Bahn, Andrew

Bai, Shi

Bai, Shuhua

Baietti, Maria Francesca

Bajek, Anna

Bakowsky, Udo

Balalaeva, Irina V.

Balanč, Bojana

Balasubramanian, Krishnan

Balcerczyk, Aneta

Ballard, David

Banciu, Manuela

Bandarenka, Hanna V.

Bandari, Suresh

Bandyopadhyay, Sulalit

Bansal, Kuldeep

Bansal, Kuldeep K.

Banti, Christina N.

Bao, Yinyin

Bao, Yongming

Baran, Anna

Barbalexis, Panagiotis

Barbosa, Carlos Mauricio

Barbosa, Raquel De Melo

Barca, Amilcare

Barelle, Caroline

Bari, Elia

Barlow, Dave

Barolo, Massimiliano

Barra, Giusi

Barradas, Thaís Nogueira

Barrett, Robert J.

Basit, Abdul

Baswan, Sudhir

Batchelor, Hannah Katharine

Battaglia, Luigi

Bauckneht, Matteo

Bax, Bridget E. 
Bayer, Wibke

Bazrafshan, Zahra

Bazwinsky-Wutschke, Ivonne

Bazylinska, Urszula

Begines Ruiz, Belén

Begines, Belen

Bello, Nicholas T.

Belvedere, Raffaella

Benhabbour, Soumya Rahima

Benito, Mónica

Benny, Ofra

Benson, Heather

Berger, Daniela Cristina

Berger, Gilles

Bergonzi, Maria Camilla

Berkó, Szilvia

Bermejo, Marival

Bernal, Eva

Bernal, Freddy Alexander

Bernardi, Simona

Berthier, Celine

Bessa, Lucinda

Bettache, Nadir

Bettini, Ruggero

Beuran, Mircea

Bhargava, Aditi

Bhatt, Priyanka

Biagi, Marco

Biagini Lopes, Luciana

Biagini, Giuseppe

Bianco, Federico

Bidarra, Sílvia J.

Bidwell, Gene

Biernasiuk, Anna

Bilgili, Ecevit

Bilia, Anna Rita

Billotey, Claire

Binda, Elisa

Bini, Marcella

Bins, Adriaan D.

Biondi, Marco

Birnbaum, Angela K.

Blaabjerg, Lasse

Blagbrough, Ian S.

Blanco-Fernandez, Barbara

Blanco-López, María Carmen

Błażewicz, Anna

Blower, Philip J.

Bobbala, Sharan

Bobis, Otilia

Bobis-Wozowicz, Sylwia
Boddu, Sai Hanumasagar

Bodea, Liviu-Gabriel

Bodenlenz, Manfred

Boekema, Bouke

Bogdan, Catalina

Boggio, Elena

Boix-Montañés, Antonio

Boldyreva, Elena

Bollati, Mariela

Bombieri, Cristina

Bonadies, Irene

Bonam, Srinivasa Reddy

Boncoeur, Emilie

Bonferoni, Maria Cristina

Bono, Nina

Bony, Badrul Alam

Borrego-Sánchez, Ana

Borska, Sylwia

Borst, Piet

Bosland, Maarten C.

Bosquillon, Cynthia

Botton, Thomas

Bouchecareilh, Marion

Boudon, Julien

Boudreau, Paul D.

Bourdon, Emmanuel

Bouropoulos, Nikolaos

Bouyer, Frederic

Boyd, Ben J.

Boyd, Chris

Braconi, Daniela

Bradley, Laura C.

Braeuer, Andreas

Brako, Francis

Brayden, David J.

Brea-Calvo, Gloria

Breier, Albert

Breitkreutz, Jörg

Brenišin, Marek

Brenza, Timothy

Briançon, Stéphanie

Brignole, Chiara

Broer, Stefan

Broude, Eugenia

Brouillet, Emmanuel

Brown, Mark A.

Brozovic, Anamaria

Brozyna, Anna

Bruni, Giovanna

Bruno, Antonino

Bryant, Christian E. 
Brzeska, Joanna

Brzezinski, Marek

Brzozowski, Tomasz

Budai-Szűcs, Mária

Bueno-Silva, Bruno

Buj-Corral, Irene

Bukowska, Joanna

Bulatovic, Maja

Bunt, Craig

Burckart, Gilbert J

Burgalassi, Susi

Burley, Jonathan C.

Busco, Giovanni

Büsselberg, Dietrich

Caballero-Casero, Noelia

Cacciatore, Ivana

Cadinoiu, Anca Niculina

Caenazzo, Luciana

Cai, Chao

Cai, Lisheng

Cai, Weimin

Caldera, Fabrizio

Calvignac, Brice

Cameron, Donald

Caminade, Anne-Marie

Cammas-Marion, Sandrine

Campana, Stefania

Campos Rosa, Joaquín María

Canals, Francesc

Canfield, Scott

Cano, Amanda

Cantore, Alessio

Cao, Xudong

Capasso, Raffaele

Capogni, Marco

Capozzi, Luigi Carlo

Caputo, Gregory

Carafa, Maria

Caramella, Carla Marcella

Carbone, Claudia

Carmona-Ribeiro, Ana M.

Carpes, Solange Teresinha

Carrasco, Elisa

Carrotta, Rita

Carter, Katharine

Caruntu, Constantin

Casalini, Tommaso

Casas-Solvas, Juan M.

Casiraghi, Antonella

Cassano, Domenico

Casteleijn, Marco
Castorena-Torres, Fabiola

Castro, Ricardo A.E.

Catalin, Bogdan

Catanzano, Ovidio

Catenacci, Laura

Catucci, Lucia

Cauda, Valentina

Causin, Paola

Cefalas, Alciviadis-Constantinos

Celakovska, Jarmila

Celebioglu, Asli

Celia, Christian

Cemazar, Maja

Ceña, Valentín

Cenciarelli, Carlo

Černý, Jiří

Cerreta, Francesca

Chacko, Jenu Varghese

Chae, Jung-woo

Chakraborty, Atanu

Challagundla, Kishore

Chamcheu, Jean Christopher

Chan, Hon Fai

Chan, Shih-Hsuan

Chandasana, Hardik

Chandrudu, Saranya

Chang, Ching-Chih

Chanishvili, Nina

Chaput, Frédéric

Chaud, Marco

Chaudhari, Smruti

Chauhan, Neeraj

Chavan, Hemantkumar

Chen, Daiqin

Chen, Hao

Chen, Haoqing

Chen, Jyh-Ping

Chen, Kelvin H.-C.

Chen, Kuo-Yu

Chen, Lin

Chen, Mei-Chin

Chen, Mingsheng

Chen, Mingzhou

Chen, Peilin

Chen, Qixian

Chen, Xinyuan

Chen, Yeong-Renn

Chen, Yuan-Chuan

Cheng, Hung-Chi

Cheng, Jya-Wei

Chereddy, Kiran 
Chetoni, Patrizia

Chiang, Chih-Sheng

Chiang, Po-Chang

Chiang, Yi-Ting

Chien, Chianshiu

Chiesa, Enrica

Chiriac, Aurica

Cho, Cheong-Weon

Cho, Dong-Woo

Cho, Hea-Young

Cho, Hyunah

Cho, Hyun-Jong

Choi, Jonghoon

Choi, Seok Ki

Chong, Li

Chong, Parskson Lee Gau

Choquesillo-Lazarte, Duane

Chou, Shih-Feng

Chow, Aviva Shing Fung

Chow, James

Chow, Michael Y.T.

Chowdhry, Dr B. Z.

Chowdhury, Ezharul Hoque

Christ, Bruno

Chrobak, Elwira

Chuang, Er-Yuan

Chuang, Yu-Chun

Chung, Suk-Jae

Chwalibóg, André

Chworos, Arkadiusz

Ciaccio, Marcello

Ciarimboli, Giuliano

Cieśla, Jolanta

Cifuentes-Rius, Anna

Cimas, Francisco J.

Cipak, Lubos

Ciszewski, Wojciech Michał

Ciura, Krzesimir

Clares, Beatriz

Cobos-Puc, Luis

Coburn, Jeannine

Coclite, Anna Maria

Codacci-Pisanelli, Giovanni

Coelho, Sílvia Castro

Cohen, Guy

Collado-González, Mar

Collins, Maurice

Colombo, Gaia

Colombo, Paolo

Cometa, Stefania

Comi, Cristoforo
Concheiro, Angel

Conejos-Sánchez, Inmaculada

Constantinescu, Cristina Ana

Conti, Bice

Conway, Barbara

Cooley, Brian C.

Corbi-Verge, Carles

Corbo, Claudia

Cordeiro, Ana Sara

Cordeiro, Rosemeyre Amaral

Córdoba, Manuel

Córdoba-Diaz, Manuel

Corvis, Yohann

Costa-Almeida, Raquel

Costabile, Gabriella

Costello, James

Cote, Christopher

Courtenay, Aaron

Coutinho, Paula

Craig, Duncan

Cristiano, Chiara

Croft, Simon

Crucho, Carina

Crucho, Carina Isabel Correia

Cruz, Juan C.

Csapo, Edit

Csiszár, Agnes

Csóka, Ildikó

Csordas, Andrew

Culot, Maxime

Cunha-Filho, Marcilio

Curtis, Anthony D.M.

Curtis, Robin

Cysewska, Karolina

Czapik, Agnieszka

D. Savić, Snežana

D'Amora, Ugo

D'Apice, Luciana

Da Pieve, Chiara

Da Silva Júnior, Arnóbio Antonio

Da Silva, A. Moreira

Da Silva, Luis Cláudio Nascimento

Da Silva, Luís Pinto

Dagmar, Merinska

Dahlgren, David

Dai, Chongshan

Dal Piaz, Fabrizio

Dalhaimer, Paul

Dalmoro, Annalisa

Dandawate, Prasad

Dangol, Manita 
Daniela, Ailincai

Daniels, Rolf

Danielsen, Michael

Dansette, Patrick M.

Dargaville, Tim

Darvin, Maxim

Das, Sudip

Dash, Ranjeet Prasad

Date, Abhijit

Datta, Kausik

Davalos, Rafael V

Dave, Rutesh

David, Sheba R.

De Araújo, Daniele Ribeiro

De Bock, Marijke

De Caro, Viviana

De Carvalho, Carla C. C. R.

De Kruijff, Robin M.

De La Mata, Javier

De Luca, Michele

De Melo-Diogo, Duarte

De Miguel, Diego

De Oliveira, Paulo Renato

De Pauw, Edwin

De Rosa, Enrica

De Rosa, Giuseppe

De Sousa, Célia Tavares

De Sousa, Frederico B.

De Villiers, Melgardt M.

De, Ranjit

De, Tanima

Dea-Ayuela, María Auxiliadora

Degoul, Françoise

Delgado, Araceli

Delgado, Daniel Ricardo

Delgado-Charro, M. Begoña

Deli, Mária A.

Démuth, Balázs

Den Haan, Joke M.M.

Desai, Diti

Desando, Giovanna

Dey, Debayan

Dhenge, Ranjit

Dhilip Kumar, Sathish Sundar

Di Girolamo, Maria

Di Liegro, Italia

Di Maro, Antimo

Di Martino, Antonio

Di Martino, Piera

Di Paolo, Antonello

Di Pasqua, Anthony J.
Di, Li

Dias, Marcos Lopes

Diat, Olivier

Digiacomo, Maria

Dijk, Frederike

Dillman, Robert O.

Dimitrov, Ivaylo

Dina, Nicoleta Elena

Ding, Jianxun

Dinica, Rodica-Mihaela

Dinu-Pirvu, Cristina-Elena

Dioguardi, Mario

Direito, Rosa

Djerada, Zoubir

Djordjević, Verica

Djuris, Jelena

Dkhar, Hedwin Kitdorlang

Długaszewska, Jolanta

Dobrzynski, Piotr

Dodi, Gianina

Dodou, Kalliopi

Domb, Avi

Dombrowski, Yvonne

Domínguez-Álvarez, Enrique

Donadio, Giuliana

Dong, Weibing

Dong, Xiaowei

Dorlo, Thomas

Dorta-Estremera, Stephanie

Douroumis, Dennis

Drabczyk, Anna

Drăgoi, Cristina Manuela

Dreesen, Erwin

Drolet, Benoît

Drozdov, Aleksey D.

Droździk, Marek

Dua, Kamal

Duan, Bin

Duan, Xiaohui

Duatti, Adriano

Dubbelboer, Ilse

Duconge, Jorge

Dudhipala, Narendar

Dugar, Rohit

Dugar, Rohit P.

Duong, Van Tu

Duran Lobato, Matilde

Durrant, Lindy G.

Duskey, Jason Thomas

Dutta, Kingshuk

Dziadas, Mariusz Tomasz 
Dzimianski, John

Dzmitruk, Volha

Dzobo, Kevin

E Serda, Rita

Edginton, Andrea N

Edwards, Vince

Eedara, Basanth Babu

Egan, Paul F

Egan, Paul F.

Egito, Eryvaldo Socrates Tabosa Do

Egorova, Ksenia

Ehrmann, Andrea

Elaissari, Abdelhamid

Eleftheriadis, Georgios

Eleonora, Russo

Elfalleh, Walid

Elhissi, Abdelbary

Elizalde, Luis E.

Elmeshad, Aliaa

Eloy, Josimar De Oliveira

El-Say, Khalid Mohamed

Else, Terry Ann

ElShaer, Amr

Endo, Makoto

Enosi Tuipulotu, Daniel

Enrione, Javier

Erdem, Emre

Erdő, Franciska

Eric Kast, Richard

Erxleben, Andrea

Escargueil, Alexandre

Escoffre, Jean-Michel

Esposito, Elisabetta

Esposito, Emilio Xavier

Espuelas, Socorro

Etrych, Tomáš

Eudald, Casals

Evans, Cameron

Facchetti, Giorgio

Fadda, Anna Maria

Fagioli, Franca

Fais, Stefano

Fakhraei Lahiji, Shayan

Fakhrullin, Rawil F.

Falsini, Sara

Faneca, Henrique

Fang, Jun

Fang, Liang

Fang, $Y i$

Fang, Yi-Ping

Fanizza, Elisabetta
Fareed, Jawed

Farhan, Mohammad

Fariña, José B.

Farkas, Attila

Fasinu, Pius

Fatouros, Dimitrios G.

Feczko, Tivadar

Feczkó, Tivadar

Federica, Foglietta

Feitosa, Eloi

Felgueiras, Helena

Feng, Sheng-Wei

Fenyvesi, Ferenc

Fernandes, Hugo

Fernandes-Cunha, Gabriella Maria

Fernández-Arévalo, Mercedes

Fernandez-Campos, Francisco

Fernandez-Garcia, Raquel

Fernández-Gutiérrez, Mar

Ferraz Gonçalves, José António

Ferreon, Allan Chris M.

Ferriz-Martínez, Roberto Augusto

Fichera, Epifanio

Fierascu, Radu Claudiu

Figueiras, Ana

Figueiredo, Isabel Vitória

Figueiredo, Marxa L.

Filho, Marcílio Cunha

Filice, Marco

Filippov, Sergey

Filova, Eva

Fini, Paola

Finke, Jan Henrik

Finsterer, Josef

Fiorica, Calogero

Firdessa, Rebuma

Fitzgerald, Seán

Flament, Marie-Pierre

Florczyk, Stephanie

Flores, Ana Isabel

Focaroli, Stefano

Fogacci, Federica

Fontana, Antonella

Fonte, Pedro

FORGET, PATRICE

Forini, Francesca $S$.

Fornaguera, Cristina

Fortuna, Ana

Forzato, Cristina

Fracasso, Giulio

Fraguas-Sánchez, Ana Isabel 
Fraix, Aurore

Francesco, Scavello

Franco, Marina Santiago

Francuzik, Wojciech

Frandsen, Stine Krog

Frank, Luiza

Frank, Luiza Abrahão

Franzè, Silvia

Freisleben, Hans-Joachim

Freitas, Vanessa Morais

Frey, Kathleen

Frizzo, Clarissa P.

Froelich, Anna

Fröhlich, Eleonore

Frolov, Andrey I.

Fromen, Cathy

$\mathrm{Fu}$, Junjie

Fuhrmann, Gregor

Fujii, Makiko

Fujinaga, Koh

Fukuta, Tatsuya

Fumoto, Shintaro

Furlani, Franco

Furneri, Pio Maria

Furuishi, Takayuki

Gabrielli, Valeria

Gabrielson, Nathan P.

Gadjanski, Ivana

Gadomska-Gajadhur, Agnieszka

Gaffet, Eric

Gala, Rikhav P.

Galateanu, Bianca

Galbis, Elsa

Galderisi, Umberto

Galelli, Luca

Gallardo, Eugénia

Gallo, Juan

Galluccio, Michele

Galstyan, Anzhela

Gaman, Mihnea-Alexandru

Ganazzoli, Fabio

Ganesan, A.

Gangadaran, Prakash

Ganguly, Samit

Gao, Chunsheng

Gao, Huile

Gao, Jin

Gao, Zhe

Garcia-Lopez, Patricia

García-Martínez, Eva

García-Mena, Jaime
García-Ramos, Juan Carlos

García-Villén, Fátima

Garello, Francesca

Garrido, María Jesus

Garriga, Rosa

Gashti, Mazeyar Parvinzadeh

Gaspar, Maria Manuela

Gáspár, Róbert

Gatzka, Martina

GAUTHIER, PASCALE

Gaviña, Pablo

Ge, Jiaoju

Gelfuso, Guilherme M.

Gendaszewska-Darmach, Edyta

Genta, Ida

Geraci, Fabiana

Gerogiorgis, Dimitrios

Geszke-Moritz, Małgorzata

Geyer, Joachim

Ghadiri, Maliheh

Ghazanfari, Lida

Ghersi, Giulio

Ghica, Mihaela Violeta

Ghica, Mihaela-Violeta

Ghiciuc, Cristina

Ghiciuc, Cristina Mihaela

Gholamipour-Shirazi, Azarmidokht

Ghonaim, Hassan

Ghori, Muhammad Usman

Ghosh, Santosh

Giansanti, Luisa

Gigliotti, Casimiro

Gikas, Evagelos

Gil, Jesus Perez

Gill, Jason

Gillespie, James W.

Giovagnoli, Stefano

Giovannelli, Pia

Giovannoni, Maria P.

Gisbert-Garzarán, Miguel

Gitlin-Domagalska, Agata

Gitsov, Ivan

Giunchedi, Paolo

Glaser, Keith

Glass, Jon

Glassman, Patrick M.

Glišić, Biljana

Gloria, Antonio

Glushakov, Alexander

Goins, William F.

Gok, Ozgul 
Gołda-Cępa, Monika

Gomes-da-Silva, Lígia C.

Gomez Cerezo, Maria Natividad

Gomez-Gil, Veronica

Gomez-Ruiz, Santiago

Gonçalves, Lídia

Gonzalez Alvarez, Isabel

González Laredo, Rubén F.

González Maglio, Daniel H.

Gonzalez, Jimena S.

González, Marta

Gonzalez-Alvarez, Isabel

Gonzalez-Alvarez, Marta

González-Nieto, Daniel

Gopalakrishnan, Gopakumar

Gordeeva, Olga

Goreham, Renee

Gorman, Greg

Gorovits, Boris

Gorzelanny, Christian

Gośliński, Tomasz

Gosselin, Ryan

Goto, Masahiro

Goto, Rieko

Goudoulas, Thomas

Goyanes, Alvaro

Gozalbes, Rafael

Gracia-Sancho, Jordi

Gradzielski, Michael

Grande, Rossella

Grassi, Mario

Grazu, Valeria

Greenhalgh, David

Grégoire, Sébastien

Grice, Jeffrey E.

Griffin, Brendan T.

Grijalvo, Santiago

Grimaudo, Maria Aurora

Grivas, Ioannis

Grivel, Jean-Claude

Grossi, Giancarlo

Grumezescu, Alexandru Mihai

Grzeskowiak, Bartosz

Gudey, Shyam Kumar

Guerrero-Martínez, Andrés

Gugliandolo, Enrico

Guillaume, Olivier

Guiot, Caterina

Gukasyan, Hovik

Gulei, Diana

Gumieniczek, Anna
Gun'ko, Vladimir M

Gunasekar, Susheel K.

Guo, Baolin

Guo, Chih-Hung

Guo, Dandan

Gupta, Prachi

Gupta, Sanjay

Gustafsson, Mats G.

Gutierrez-Aguilar, Manuel

Guzmán-Navarro, Manuel

Guzow-Krzemińska, Beata

Ha, Eun-Sol

Haas, Heinrich

Haddrell, Allen E.

Hadinoto, Kunn

Haemmerich, Dieter

Hageman, Michael

Hagenbuch, Bruno

Hagesaether, Ellen

Hama, Susumu

Hamman, Joias

Han, Felicity

Han, Sifei

Han, Xiuguo

Han, Yingchao

Hanafy, Nemany A. N.

Hanawa, Takehisa

Handral, Harish

Hanieh, Patrizia Nadia

Hann, Hie-Won

Hansen, Niels

Hanson, Ben

Hao, Jinsong

Hardies, Stephen C.

Hardtke-Wolenski, Matthias

Hardy, John G.

Harker, Anthony

Haron, Mona

Hasan, Abshar

Hasanzadeh Kafshgari, Morteza

Hasegawa, Urara

Hashidzume, Akihito

Hashimoto, Michinao

Hatahet, Taher

Hathout, Rania

Hathout, Rania Mohammed

Hatziantoniou, Sophia

Haware, Rahul V.

Hawcutt, Daniel B.

Hawthorne, Susan

Hayashida, Morihiro 
He, Hongliang

$\mathrm{He}$, Jing

$\mathrm{He}$, Mei

He, Mingyu

He, Weilue

Healy, Anne Marie

Heard, Charles

Heath, Timothy

Heger, Zbynĕk

Heiduschka, Peter

Heinämäki, Jyrki Tapio

Hellstrom, Per

Hémadi, Miryana

Hendriks, Hans H.

Hens, Bart

Hering-Smith, Kathleen

Herraez, Elisa

Hertzler, Russell

Heskamp, Sandra

Hessel-Pras, Stefanie

Hildreth, Blake E.

Hilgendorf, Constanze

Hinrichs, Wouter L. J.

Hiratsuka, Masahiro

Hirayama, Fumitoshi

Hiroaki, Hidekazu

Hirose, Ryohei

Hirschberg, Cosima

Ho, Tsing-Fen

Hoeben, Rob

Hoffmann, Klaus H.

Hofmann, Ute

Hollmann, Axel

Holst, Helle

Homem-de-Mello, Mauricio

Hooper, Douglas C.

Horhat, Florin George

Hori, Akiko

Hornebecq, Virginie

Horowitz, John D.

Hortelano, Gonzalo

Hoskins, Clare

Hossain, A. S. M. Monjur Al

Howard, Erin W.

Howes, Philip

Hsia, Shih-Min

$\mathrm{Hu}$, Jack

$\mathrm{Hu}$, Shang-Hsiu

$\mathrm{Hu}$, Teh-Min

$\mathrm{Hu}$, Xianglong

Hua, Kuo-Feng
Hua, Xiaoqing

Huang, Jiangeng

Huang, Wei

Huang, Yuanyu

Huang, Yugang

Huang, Yunlong

Huerta-Angeles, Gloria

Huhtinen, Mirja K.

Hui, Patrick C.L.

Huntosova, Veronika

Hurtig, Mark

Hutchins, Kristin M.

Huth, Sebastian

Hwang, Mintai

Hwang, Seung Rim

Hwang, Sung-Joo

Iaccino, Enrico

Iacone, Roberto

Iannuccelli, Valentina

Iatridi, Zacharoula

Iatrou, Hermis

Ibrahim, Sherif Abdelaziz

Ibrahim, Toni

Iglesias, Bernardo

Ignat, Maria

Ignjatovic, Nenad

Ikushiro, Shin-ichi

Ilaria, Ortensia

Ilhan, Harun

Ilic-Stojanovic, Snezana

Ilies, Marc A.

Ilyinskii, Petr O.

Im, Chang-Nim

Imberti, Cinzia

Imperatore, Roberta

Inbraj, Stephen

Infante, Arantza

Ingelsson, Martin

Innamorati, Giulio

Inoue, Daisuke

Ionescu, Mihaela Ileana

Ionita, Petre

Iorio, Ronald M.

Iqbal, Hafiz. M. N

Iqbal, Shoaib

Irie, Tetsumi

Irwin, David C.

Isola, Gaetano

Israel, Liron Limor

Isreb, Abdullah

Issa, Michele Georges 
Ita, Kevin

Ito, Shingo

Iwasaki, Takashi

Iwasaki, Yoshiaki

J. Storkus, Walter

Jagusiak, Anna

Jain, Akshay

Jaiswal, Jagdish Kumar

Jaiswal, Jyoti K.

Jakimovski, Dejan

Jakubowski, Witold

Jammalamadaka, Udayabhanu

Jamwal, Rohitash

Janagam, Dileep R.

Janagam, Dileep Reddy

Janek, Tomasz

Jang, Keon-Soo

Jang, Mi-Kyeong

Janicka, Małgorzata

Janjic, Jelena M.

Janoušková, Olga

Jaramillo-Flores, Maria

Jaromin, Anna

Jasminder, Sahi

Jaszcz, Katarzyna

Jedelská, Jarmila

Jee, Jun-Pil

Jee, Shiouhwa

Jelonek, Katarzyna

Jelsch, Christian

Jencova, Vera

Jenkins, Samir V.

Jensen, Svend Borup

Jeon, Jongho

Jeong, Hyun Young

Jeong, Jun-ho

Jeong, Seong Hoon

Jiang, Chengmin

Jiang, Yanbin

Jiang, Ziwen

Jianliang, Shen

Jicsinszky, László

Jin, Jun-O

Jin, Sunggiu

Jin, Xin

Jindřich, Jindřich

Jing, Hui

Jo, Yeon-Ji

Jones, Marjorie

Joshi, Aditya

Joshi, Sameer
Jović, Danica

Joyce, Paul

Józefczak, Arkadiusz

Juang, Yi-Je

Juarez, Karla

Juhasz, Kata

Juif, Pierre Eric

Juluri, Abhishek

Jung, Hyungil

Jung, Jae Hwan

Jung, Seunho

Jung, Yunjin

Jungsuwadee, Paiboon

Junsong, Li

Juppo, Anne

Kabała-Dzik, Agata

Kachrimanis, Kyriakos

Kaczmarek, Beata

Kadam, Avinash

Kalhapure, Rahul S.

Kalia, Yogeshvar N.

Kalló, Gergő

Kalyan, Ramesh

Kamada, Kai

Kambayashi, Atsushi

Kambhampati, Siva Pramodh

Kamihira, Masamichi

Kaminski, Piotr

Kamiński, Tomasz

Kanada, Masamitsu

Kang, Han Chang

Kang, Hee Eun

Kang, Myung Joo

Kang, Nae Gyu

Kang, Yunqing (Kevin)

Kankala, Ranjith Kumar

Kanugula, Anantha Koteswararao

Kao, Chai-Lin

Kao, Li-Ting

Karalis, Vangelis D.

Karamouzis, Michalis

Karashima, Masatoshi

Karazniewicz, Marta

Karbownik, Agnieszka

Karcz, Dariusz

Kardas, Przemyslaw

Karewicz, Anna

Karim, Nasiara

Karuppan, Mohan Kumar Muthu

Kasperczyk, Janusz

Kasperek, Regina 
Kasprzak, Artur

Kastellorizios, Michail

Katarzyna, Malarz

Kathuria, Himanshu

Katona, Gábor

Kaushik, Neha

Kauss, Tina

Kawakami, Kohsaku

Kawakami, Shigeru

Kawano, Kumi

Kazakov, Sergey

Kelarakis, Antonios

Kepinska, Marta

Kerr, Ian

Kertmen, Ahmet

Kesharwani, Prashant

Kesharwani, Siddharth

Kesharwani, Siddharth S.

Khalf, Abdurizzagh

Khan, Omar F.

Khandavilli, Udaya

Khilwani, Rakesh

Khoder, Mouhamad

Khudyakov, Igor $\mathrm{V}$

Khung, Yit Lung

Khurana, Varun

Khurshid, Zohaib

Khutoryanskiy, Vitaliy

Kijenska, Ewa

Kim, Dong Wuk

Kim, Il Won

Kim, Jeong-Hwan

Kim, Jin-Seok

Kim, Jinwook

Kim, Keun-Sik

Kim, Ki-Taek

Kim, Kwanoh

Kim, Min-Soo

Kim, Ok-Kyung

Kim, Seong-Gon

Kim, Sung-Kun (Sean)

Kim, Yeu-Chun

Kinaret, Pia

Kirby, Daniel

Kirton, Stewart B.

Kiselev, Anton

Kittel, Agnes

Kitzmüller, Claudia

Klajnert-Maculewicz, Barbara

Klang, Victoria

Klar, Agnes
Kleinebudde, Peter

Kluczyk, Alicja

Knapik-Kowalczuk, Justyna

Knezevic, Nikola

Ko, Eun-ju

Kobuchi, Shinji

Kodidela, Sunitha

Koga, Yoshikatsu

Köhler, Karsten

Kojima, Chie

Kolanjiyil, Arun Varghese

Kolar, Mitja

Kolisek, Martin

Kolmar, Harald

Kolobov, Alexandr Alexandrovich

Kołodziejczyk-Czepas, Joanna

Kolosnjaj-Tabi, Jelena

Kondo, Hiromu

Kong, L.X.

König, Jörg

Konjufca, Vjollca

Konstantinov, Spiro M.

Kontogiannidou, Eleni

Kontoravdi, Cleo

Koo, Hee-beom

Koolivand, Abdollah

Kootala, Sujit

Kopecki, Zlatko

Kopel, Pavel

Korchowiec, Beata

Korkmaz, Emrullah

Koroleva, Marina Yu

Korupalli, Chiranjeevi

Korzhikova-Vlakh, Evgenia

Korzhikov-Vlakh, Viktor

Kosicka-Noworzyń, Katarzyna

Kostić, Aleksandar Ž.

Kostka, Libor

Kotlyarova, Anastasiia

Kotyla, Przemyslaw J.

Kovacs, Anita

Kozak, Maciej

Kozielski, Lucjan

Koziolek, Mirko

Kozłowska, Joanna

Kozlowska, Justyna

Kragović, Milan

Krajczewski, Jan

Krasnikov, Boris

Krishnan, Vinu

Kruger, Cherie 
Krüger, Marcus

Krupa, Anna

Kubiak, Katarzyna

Kubo, Anna-Liisa

Kubo, Yoshiharu

Kucuk, Israfil

Kuddannaya, Shreyas

Kuehl, Philip J.

Kuentz, Martin

Kulbacka, Julita

Kulkarni, Ketav

Kumar K. B., Vinaya

Kumar Patel, Sravan

Kumar, Janani

Kumar, Mukesh

Kumar, Pradeep

Kumar, Raj

Kumar, Ramesh

Kumar, Samir

Kumar, Vineet

Kumeria, Tushar

Kunda, Nitesh

Kung, Chin-Ping

Kuo, Yao-Haur

Kurek, Mateusz

Kurganov, Boris

Kurrikoff, Kaido

Kusamori, Kosuke

Kushwah, Varun

Kwiatkowski, Pawel

Kwilas, Steven

Kwok, Henry Hang Fai

Kwon, Il-Keun

Kwon, Tae-Hyuk

Kwon, Young M.

Ladyzynski, Piotr

Lahiji, Shayan Fakhraei

Lahiri, Tanaya

Lai, Chian-Hui

Lai, Francesco

Lai, Jui-Yang

Lakshminarayanan, Rajamani

Lalatsa, Aikaterini

Lalic-Popovic, Mladena

Langner, Marek

Lankoff, Anna

Lannoy, Damien

Lanza, Marco

Lanza, Michele

Lapteva, Maria

Laquintana, Valentino
Largent-Milnes, Tally

Larhrib, Hassan

Lasagni, Laura

Latif, Muhammad

Lau, Wai Man Raymond

Laurini, Erik

Lauro, Maria Rosaria

Lauzon, Marc-Antoine

Lazzara, Giuseppe

Le Gall, Tony

Le, Hoang-Thanh

Lebeau, Luc

Lebrón, José Antonio

Leclair, Gregoire

Leclerc, Denis

Lee, Aeju

Lee, A-W

Lee, Beom-Jin

Lee, Chia-Hung

Lee, Heung Kyu

Lee, Hong-Ki

Lee, Hye Suk

Lee, Jaehwi

Lee, Jangik Ike

Lee, Jeongmi

Lee, Jong Bong

Lee, Jung Seok

Lee, Sangkil

Lee, $\mathrm{Tu}$

Lee, Wonhye

Lee, Yi-Chih

Lee, Yongbok

Lee, Yonghyun

LEGRAND, François-Xavier

Lehr, Thorsten

Lei, Wei

Leitner, Wolfgang $\mathrm{W}$

Lemmerer, Andreas

Leporatti, Stefano

Leppert, Wojciech

Lesnikowski, Zbigniew J.

Lesuisse, Dominique

Lesyk, Roman B.

Leung, Ada W.Y.

Leung, Shui Yee

Levina, Aviva

Lewandowska, Katarzyna

Lewis, Gladius

Leyssens, Tom

Leyva-Gómez, Gerardo

Li, Chuan 
Li, Feng
Li, Fuhai
Li, Guangdi
Li, Jason
Li, Jie
Li, Jinyao
Li, Junjie
Li, Li
Li, Liping
Li, Meng
Li, Na
Li, S. Kevin
Li, Wen-Wu
Li, Xiaohong
Li, Xue
Li, Yang
Li, Yunfei
Li, Zhiyu
Liao, Ai-Ho

Liao, Yonghong

Libra, Massimo

Lietzau, Grazyna

Lim, Eun-Kyung

Lim, Jason

Lima, Sofia

Lin, Haishu

Lin, Hai-Shu

Lin, Hsiu-Mei

Lin, Peter P.

Lin, T. H

Lin, Tung-Yi

Lin, Wei-Chao

Lin, Weifeng

Lin, Wenjing

Lin, Yang

Lin, Zhoumeng

Ling, Chen

Lipke, Peter

Lis Arias, Manuel J.

Liu, Chao-Lin

Liu, Cheuk Lun

Liu, Der-zen

Liu, Fang

Liu, Fei

Liu, Li

Liu, Ting-Yu

Liu, Yu

Liu, Yun-Jun

Liu, Zhi

Lobo, Glenn P.

Lochhead, Jeffrey J.
Lollo, Giovanna

Long, Chiau Ming

Longo, Joao Paulo

Lönnberg, Harri

Lopalco, Antonio

Lopedota, Angela

Lopes, Carla Martins

López Castellano, Alicia

Lopez-Camarillo, Cesar

López-Camarillo, César

Lopez-Cara, Luisa Carlota

López-Cornejo, Pilar

López-López, Manuel

Lorberboum-Galski, Haya

Loretz, Brigitta

Loureiro, Joana A.

Lozano, Jose Manuel

$\mathrm{Lu}$, Ming

Lu, Weiyue

$\mathrm{Lu}, \mathrm{Yi}$

Lucaciu, Constantin Mihai

Lucas, Andrew T.

Lucas, Guilherme

Lucchetti, Donatella

Luciani, Paola

Lucio, Victor

Luckham, Paul

Luebbert, Christian

Lugović-Mihić, Liborija

Lukyanov, Pavel A.

Luliński, Piotr

Lunter, Dominique

Luo, Kui

Luo, Rongcong

Luppi, Barbara

Lusi, Matteo

Lux, Jacques

Luzardo-Álvarez, Asteria

Luzi, Francesca

M. Caramella, Carla

$\mathrm{Ma}$, Jun

Ma, Xiang

Maayah, Zaid

Machado-Aranda, David

Machelart, Arnaud

Madheswaran, Thiagarajan

Madhyastha, Harishkumar

Madka, Venkateshwar

Maeda, Kazuya

Maeda, Tomoki

Maeng, Han-Joo 
Maestrelli, Francesca

Maggio, Rubén M.

Magnani, Mauro

Magri, Giulia

Maier, Jeanette A.M

Mair, Lamar O.

Maisel, Katharina

Majkowska-Pilip, Agnieszka

Majolino, Domenico

Major, Ian

Majumder, Aniruddha

Majumder, Poulami

Makarska-Bialokoz, Magdalena

Malcolm, Karl

Maleki, Hajar

Malik, Adeel

Malik, Paul R. V.

Malik, Ravinder

Malinge, Jean-Marc

Mallandrich, Mireia

Malo De Molina, Paula

Malzert-Fréon, Aurélie

Mamidi, Narmidi

Manakhov, Anton

Manca, Maria Letizia

Manconi, Maria

Mandal, Abhirup

Manetti, Dina

Manfredini, Stefano

Mangal, Sharad

Mangas, Victor

Mangino, Giorgio

Maniruzzaman, Mohammed

Manna, Saikat

Mansi, Rosalba

Mao, Qingcheng

Marcos-Contreras, Oscar A

Marei, Hany

Mareș, Mihai

Margreitter, Christian

Marín Ramos, Nagore Isabel

Marin, Luminita

Marino Gammazza, Antonella

Markl, Daniel

Markowicz-Piasecka, Magdalena

Maroni, Alessandra

Marová, Ivana

Marradi, Marco

Martellucci, Stefano

Martín Rengel, Antonio E.

Martin, Francisco
Martin, Maria Alba

Martín-Aragón, Sagrario

Martinez Alonso, Marta

Martinez Rodriguez, Fleming

Martinez, Cristina

Martinez, Vicente

Martínez-Carmona, Marina

Martínez-García, Marcos

Martínez-López, Ana Luisa

Martino, Sabata

Martins, Alessandro F.

Martins, José A.

Martins, Natália

Marto, Joana

Marverti, Gaetano

Marwitz, Sebastian

Marzano, Cristina

Masalova, Olga V.

Mas-Bargues, Cristina

Mashima, Ryuichi

Mashruwala, Ameya

Maslov, Mikhail A.

Massa, Antonio

Mastinu, Andrea

Mastrotto, Francesca

Masuda, Takeshi

Matei, Cristian

Matencio, Adrián

Matijašić, Gordana

Matikonda, Siddharth

MATRICARDI, Pietro

Matsugaki, Aira

Matsui, Reiko

Matsumoto, Yasuhiko

Matsunaga, Kazuhisa

Matsuura, Bunzo

Matta, Murali Krishna

Mattei, Vincenzo

Matters, Gail L.

Matthiesen, Rune

Matys, Jacek

Mauri, Emanuele

Mayer, Christian

Mazel, Vincent

Mazet, Roseline

Mazzaglia, Antonino

Mazzoni, Chiara

Mcardle, Patrick

McArdle, Stephanie

McCartney, Fiona

McCauley, Heather A 
McConville, Christopher

McConville, Jason T.

Mcdonald, Peter

McDowell, Arlene

McHugh, Kevin

McMurry, Jonathan

$\mathrm{Md}$, Shadab

Medarevic, Djordje

Medeiros, Ana Cláudia D.

Medina Juarez, Luis Angel

Medina Velázquez, Luis Alberto

Medina-Ramírez, Iliana

Mees, Maarten

Mei, Ya-Fang

Meier, Robert J.

Melero, Ana

Melo-Diogo, Duarte De

Mendonça-Lima, Leila

Mendoza-Muñoz, Néstor

Mendyk, Aleksander

Menghini, Luigi

Merino, Sonia

Merlino, Francesco

Mestres, Conxita

Meyer, Florent

Mező, Gábor

Mezzetta, Andrea

$\mathrm{Mi}$, Fwu-Long

Mi, Lan

Micek, Agnieszka

Mielańczyk, Anna

Mihai, Mara

Mijovic, Budimir

Milasius, Rimvydas

Milavetz, Barry

Milazzo, Mario

Mildner, Michael

Milewska, Maria J

Miller, Ian Steuart

Miller, Larry

Minami, Masaaki

Minghetti, Paola

Mircioiu, Constantin

Mirza, Sabiruddin

Mishra, Pawan Kumar

Mitchell, Andrew

Miura, Yoshiko

Miyake, Masateru

Miyata, Yoshihiko

Miyata, Yoshiki

Miyoshi, Norio
Mlera, Luwanika

Mochizuki, Shinichi

Modi, Nisarg

Mohammed, Yousuf

Moiseev, Roman V.

Mollapour, Mehdi

Momin, Mohammad Abdul Motalib

Mondal, Goutam

Mondal, Sudip

Montanari, Elita

Monteiro, Carlos

Monteiro, Cláudia

Montenegro, Lucia

Monti, Daniela

Moon, Cheol

Moon, Geon Dae

Mooranian, Armin

Mora, Claudia

Morales, Javier

Morales-Rojas, Hugo

Morbidelli, Lucia

Moreau, Regis

Morefield, Garry

Morgado, Jorge

Morgat, Clément

Morgen, Michael

Moritz, Michał

Mornar, Ana

Morotomi, Takahiko

Morris, Gordon

Morris, John B.

Mosqueira, Matias

Mosqueira, Vanessa Carla Furtado

Moss, Gary

Mourtas, Spyridon

Mouzakis, Dionysios E.

Moyano-Mendez, Jose Ramon

Mracek, Tomas

$\mathrm{Mu}$, Qingxin

Muchowicz, Angelika

Muchtaridi, Muchtaridi

Muenzberg, Michael

Mujtaba, Muhammad

Mukherjee, Dwaipayan

Mukherjee, Raj

Mukherjee, Sudip

Muller, Christian

Mundra, Vaibhav

Muntean, Dana Maria

Muntimadugu, Eameema

Murab, Sumit 
Murakami, Teruo

Murnane, Kevin

Musazzi, Umberto M.

Musuc, Adina Magdalena

Musumeci, Domenica

Musumeci, Teresa

Muthu Karuppan, Mohan Kumar

Muthuraju, Sangu

Muthusamy, Nagendran

Muzykantov, Vladimir R.

Muzzalupo, Rita

Myc, Andrzej

Myers, Alan Lewis

$\mathrm{Na}$, Dong Hee

$\mathrm{Na}$, Young-Guk

Nag, Okhil K.

Nagai, Noriaki

Nagata, Toshi

Nair, Anroop B.

Nair, Devatha P.

Nair, Praful R.

Nair, Pramod

Najlah, Mohammad

Nakai, Hiroyuki

Nakamura, Kazufumi

Nakanishi, Takeo

Nakano, Masahito

Nakielski, Pawel

NAM, Ki Chang

Namgaladze, Dmitry

Namjoshi, Sarika

Nanaki, Stavroula

Nanavati, Charvi

Nanjundan, Meera

Narayan, Mahesh

Narayanasamy, Suresh

Narayanaswami, Vasanthy

Nartowski, Karol P.

Natesan, Shanmugasundaram

Navarrete, Paola

Navarro, Estanis

Navarro-Ruiz, Andrés

Navran, Arash

Nawaz, Muhammad

Nayak, Usha Y.

Naylor, Andrew

Negri, Donatella

Nethi, Susheel Kumar

Neubert, Reinhard

Neufurth, Meik

Neves, Vera
$\mathrm{Ng}$, Keng Wooi

Ngen, Ethel J.

$\mathrm{Ni}$, Rong

Nicolau, David

Nicoli, Sara

Nielsen, Line Hagner

Niemczyk, Agata

Niemirowicz-Laskowska, Katarzyna

Nightingale, Adrian

Nikolakakis, Ioannis

Nikolett, Kállai

Nishida, Koyo

Nita, Loredana E.

Noh, Keumhan

Nokhodchi, Ali

Noma, Hidetaka

Nopens, Ingmar

Nosoudi, Nasim

Notario-Pérez, Fernando

Novikov, Andrei

Novinec, Marko

Nsooudi, Nasim Nsooudi

Numez, Estrella

Nurunnabi, Md

Nuxoll, Eric

O'Connor, José Enrique

O'Shaughnessy, Ryan

Obata, Yasuko

Obeng, Samuel

Obuobi, Sybil Akua Okyerewa

Ochowiak, Marek

Ogawa, Kazuma

Oh, Daniel

Oh, In-Jae

Oh, Ji-Hyeon

Oh, Kyung Taek

Ojha, Shreesh K.

Oka, Sarang

Okamoto, Motoki

Okamura, Yosuke

Okesola, Babatunde

Okuda, Tomoyuki

Olaru, Octavian Tudorel

Olaso, Gloria

Olczyk, Paweł

Olewnik-Kruszkowska, Ewa

Oliveira, David De

Oliveira, J.Vladimir

Oltolina, Francesca

Omasa, Takeshi

Omote, Hiroshi 
Onishi, Hiraku

Ordaz-Ortiz, José Juan

Orlando, Tomas

Orlova, Anna

Ortega, Paula

Osada-Oka, Mayuko

Ostacolo, Carmine

Østergaard, Jesper

Ostos Marcos, Francisco José

Ostrovsky, Olga

Oswald, Stefan

Osyczka, Anna Maria

Ota, Satoshi

Otero, Carolina

Otero-Espinar, Francisco Javier

Otsuka, Makoto

Otte, Andrew D.

Otto, Daniel P.

Ouro, Alberto

Ouyang, Defang

Ouyang, Xiao-kun

Oya, Yuichi

Ozawa, Shogo

Ozsvari, Bela

Pacifici, Roberta

Padrela, Luis

Paduch, Roman

Pagano, Cinzia

Page, Susanne

Paine, Stuart

Paixão, Paulo

Pal, Dhananjay

Palamà, Ilaria

Pall, Emoke

Palmiero, Umberto Capasso

Palugan, Luca

Pan, Guoqin

Pan, Guoqing

Pan, Guoyu

Pan, Jiayi

Panayiotou, Costas

Panczyk, Tomasz

Pandey, Amit V

Pangeni, Rudra

Panikkanvalappil, Sajanlal R.

Pant, Bishweshwar

Panzarini, Elisa

Paolino, Donatella

Papadopoulos, Dimitrios

Papadopoulou, Lefkothea

Papakyriakou, Athanasios
Papini, Emanuele

Papke, Roger L.

Paquette, Benoit

Paramythiotou, Elizabeth

Parashar, Deepak

Parayath, Neha

Parayath, Neha N.

Pardi, Norbert

Paris, Juan

Park, Chulhun

Park, Frank

Park, Hwan-Woo

Park, Jeong-Sook

Park, Jun Beom

Park, Jung-Hwan

Park, Ki Soo

Park, Kinam

Park, Kyung Min

Park, Wooram

Parojčić, Jelena

Parrish, Richard

Passaes, Caroline Pereira Bittencourt

Passerini, Nadia

Pastor, Fernando

Pastor-Anglada, Marçal

Patel, Ketan

Patil, Veerupaxagouda

Patterson, Adam V.

Patterson, Jennifer

Paudel, Raj

Paulusse, Jos

Pautz, Andrea

Pavliňáková, Veronika

Pavlos, Klepetsanis

Pavlovic, Nebojsa

Pawlik, Andrzej

Pedrosa, Pedro

Peeters, Elisabeth

Peeters, Marloes

Peinado, María Ángeles

Peláez, Rafael

Pellegatta, Serena

Peltonen, Leena

Peluso, Ilaria

Peña Fernández, María Ángeles

Penchev, Hristo

Penfornis, Patrice

Peng, Lihong

Peptu, Cristian

Perazzoli, Gloria

Perche, Federico 
Perche, Fédérico

Perduca, Massimiliano

Pereira, Ruben

Pereira-Leite, Catarina

Pérez De Val, Bernat

Perez-Alvarez, L.

Pérez-Gracia, María-Teresa

Perinelli, Diego Romano

Perioli, Luana

Peris, José-Esteban

Perissutti, Beatrice

Perrone, Anna Myriam

Perteghella, Sara

Perugini, Paola

Peruzynska, Magdalena

Petcu, Cristian

Petrova, Guenka

Petrović, Lidija

Peviani, Marco

Piacentini, Emma

Piao, Xijun

Piatkevich, Kiryl

Piato, Angelo

Piedade, Ana Paula

Pierdomenico, Johnny Di

Pilicheva, Bissera

Pilli, Nageswara

Piluzza, Giannella

Pindelska, Edyta

Pinheiro, Lídia

Pini, Luigi Alberto

Pinnapireddy, Shashank Reddy

Pinteala, Mariana

Pinto, Eugénia

Pinto, Joao

Pinto, Sandra

Piquette-Miller, Micheline

Piras, Anna Maria

Pisaneschi, Federica

Piscioneri, Antonella

Pishnamazi, Mahboubeh

Pissarek, Margit

Pitsikalis, Marinos

Pitt, Kendal

Pitterl, Florian

Pitto-Barry, Anaiis

Piva, Terrence

Planinšek, Odon

Płaza, Grażyna

Ploetz, Evelyn

Plotniece, Aiva
Poeggeler, Burkhard

Poggi, Alessandro

Pohl, Carolina H.

Pohlmann, Adriana

Pokorny, Marek

Polakowski, Nicholas J.

Polgarova, Kamila

Pollet, Jeroen

Polo-Parada, Luis

Polyakov, Nikolay

Poma, Alessandro

Pomel, Sebastien

Popa, Lacramioara

Popat, Amirali

Porollo, Aleksey

Porter, Christopher J. H.

Poŝa, Mihalj

Post, Janine

Potrich, Cristina

Pouliopoulos, Antonis

Pourbaghi Masouleh, Milad

Prabha, Swayam

Pradhan, Shankali

Pramod, Kannissery

Pranjol, Zahid

Prashar, Sanjiv

Prassl, Ruth

Prat, Maria

Préat, Véronique

Prestidge, Clive A.

Prévostel, Corinne

Pricl, Sabrina

Priola, Emanuele

Privette Vinnedge, Lisa

Prohens, Rafel

Przybyłek, Maciej

Puchkova, Ludmila V.

Puglia, Carmelo

Puiggalí-Jou, Anna

Punzo, Francesco

Putra, Okky Dwichandra

Putrinš, Marta

Qi, Feng

Qi, Zhou

Qin, Xiaoli

Qiu, Mingfeng

Qu, Xiaozhong

Quagliariello, Vincenzo

Quan, Guilan

Quiñones, Luis Abel

Quintanar-Guerrero, David 
Quintavalla, Arianna

Quodbach, Julian

R. El-Seedi, Hesham

Rabanal Anglada, Francesc

Radchenko, Eugene V.

Radeghieri, Annalisa

Rafael, Diana

Ragno, Gaetano

Ragusa, Andrea

Rahman, Khondaker Miraz

Rai, Akhilesh

Rai, Ankit

Rai, Prakash

Raimi-Abraham, Bahijja

Rais, Rana

Rajendran, Jagathesh Chandra

Rajendran, Naresh Kumar

Rajić, Zrinka

Rallis, Michail

Ramalingam, Prakash

Ramasamy, Thiruganesh

Ramos Cabrer, Pedro

Ramsköld, Daniel

Rancan, Fiorenza

Ranganathan, Srivathsan

Rangger, Christine

Ranjan, Amalendu P.

Ranjan, Ashish

Rapeanu, Gabriela

Rasskazov, Ilia

Rathinasabapathy, Anandharajan

Rau, Ileana

Rauckhorst, Adam

Rautio, Jarkko

Rauwel, Protima

Ravaioli, Stefano

Ravula, Abhigyan

Regdon, Géza

Reis, Catarina

Reis, Catarina Pinto

Reisfeld, Brad

Reissmann, Siegmund

Reker, Daniel

Remedios Serrano, Dolores

Ren, Yong

Rengasamy, Kannan

Renò, Filippo

Renu, Sankar

Renukuntla, Jwala

Repka, Michael

Reverter, Enric
Rho, Hoon Suk

Riau, Andri Kartasasmita

Ribeiro, Antonio

Ribeiro, Helena

Ribeiro, Helena Margarida

Ribeiro, Viviana P.

Ricci, Maurizio

Richard, Cyrille

Richards, Dylan J.

Richards, Dylan Jack

Richardson, Alan

Richardson, Joseph

Rinaldi, Federica

Rinnerthaler, Mark

Ripoli, Cristian

Ristori, Sandra

Rizvanov, Albert

Rizzi, Rosanna

Rizzo, Fabio

Rizzolio, Flavio

Roberts, Arthur G.

Robinson, Nirmal

Rocha, João

Rochfort, Keith

Rodallec, Anne

Roderfeld, Martin

Rodilla, Vicent

Rodrigues, João

Rodriguez, Luis

Rodríguez-Hornedo, Naír

Roggo, Yves

Rogobete, Alexandru Florin

Rom, Slava

Romano, Giovanni Luca

Romański, Michał

Rombolà, Laura

Romerio, Fabio

Romero-Uribe, Gabriela

Romiti, Giulio Francesco

Ronsard, Larance

Roohnikan, Mahdi

Rosa, Arianna Carolina

Rosado, Catarina

Rosales-Hernández, Martha Cecilia

Rosania, Gustavo

Roseanu, Anca

Rosenholm, Jesicca

Roseti, Livia

Rosière, Rémi

Rossi, Alessandra

Rossi, Filomena 
Rossi, Silvia

Rosso, Natalia

Roszkowska, Anna

Rothe, Thomas

Rotondo, John Cahrles

Rotstein, Coleman

Roullin, V. Gaëlle

Rozsival, Pavel

Rozza, Ariane

Rubilotta, Emanuele

Rubino, Ilaria

Rubio Alonso, Juan

Ruda-Kucerova, Jana

Rudd, Sean G

Rudra, Arnab

Ruiz, Amalia

Ruiz-Caro, Roberto

Ruiz-Ederra, Javier

Rusciano, Dario

Russo, Paola

Ruzza, Chiara

Rybarczyk-Pirek, Agnieszka J.

Rybka, Jakub Dalibor

Rychahou, Piotr

Ryu, Ji Hyun

Ryu, Jung Su

Sa-Barreto, Livia L.

Sabatino, David

Sachar, Madhav

Sacher, Stephan

Sack, Ulrich

Sah, HongKee

Sahota, Tarsem S.

Saint-Pol, Julien

Salamanca, Constain $\mathrm{H}$.

Salameh, Chrystelle

Salaün, Fabien

Saleem, Imran

Saleh, Tawfik

Salhab, Hassan

Saliev, Timur

Salmina, Alla

Salunke, Smita

Samal, Sangram

Samanta, Subarna

Sambuy, Yula

Samson, Abraham O.

Sánchez Navarro, Amparo

Sanchez, Elena

Sánchez-García, David

Sandoval-Yáñez, Claudia
Sandri, Giuseppina

Sands, Jeff

Sansone, Francesca

Santi, Melissa

Santi, Patrizia

Santos, Adriana O.

Santos, Cleydson Breno R.

Santos, Miguel

Santos-Coquillat, Ana

Sanz-Clemente, Antonio

Sapino, Simona

Sapudom, Jiranuwat

Sara, Zalba

Saravanakumar, Kandasamy

Sarli, Vasiliki

Sarraguça, Mafalda

Sarsour, Ehab

Sasai, Yasushi

Šatkauskas, Saulius

Sato, Hideyuki

Sato, Kazuhide

Sato, Shintaro

Sato, Shuzo

Sattler, Wolfgang

Sautto, Giuseppe A.

Sawada, Kazuhiko

Sayers, Edward J

Sazonova, Margarita A.

Ščasný, Milan

Schaiquevich, Paula

Schlereth, Simona L.

Schlich, Michele

Schlindwein, Walkiria

Schmidl, Doreen

Schmutz, Marc

Schnieke, Angelika E.

Schols, Dominique

Scholz, Carmen

Schönthal, Axel H.

Schulz, Florian

Schumann, Canan

Schwaminger, Sebastian

Scognamiglio, Pasqualina Liana

Scott, Christopher

Segale, Lorena

Seifert, Gotthard

Seiler, Magdalene J.

Selbo, Pål Kristian

Selin, Victor

Selwood, David L.

Seo, Ho Seong 
Serda, Maciej

Serpe, Loredana

Serrano, Dolores R.

Seto, Yoshiki

Settimio, Pacelli

Sgarbi, Gianluca

Shabatina, Tatyana

Shah, Dhaval K.

Shahjamali, Mohammad

Shaik, Anver Basha

Shakeel, Faiyaz

Shakibaei, Mehdi

Shakya, Akhilesh

Shamieh, Said

Shankar, Eswar

Sharbeen, George

Shariare, Mohammad

Sharma, Prashant

Shavandi, Amin

Shcharbin, Dzmitry

Sheikh Mohamed, $\mathrm{M}$.

Shen, Hsin-Hui

Shen, Jie

Shen, Zhiqiang

Sheng, Ruilong

Shevtsov, Maxim

Shi, Lingyan

Shi, Wen

Shibli, Jamil Awad

Shimizu, Taro

Shimizu, Tatsuya

Shimodaira, Shigetaka

Shimpi, Manishkumar

Shin, Beom Soo

Shin, Crystal S.

Shin, Meong Cheol

Shin, Soyoung

Shirasaka, Yoshiyuki

Shirazian, Saeed

Shugo, Suzuki

Shukla, Snehal K.

$\mathrm{Si}, \mathrm{Ke}$

Siafaka, Panoraia

Siamidi, Angeliki

Sideratou, Zili

Sidorenko, Viktoriya S.

Sidoti, Antonina

Siegmund, Werner

Sieni, Elisabetta

Signore, Giovanni

Sikora, Mariusz
Silva Filho, Edson

Silva, Ana Catarina

Silva, Joana

Silva, Nuno Helder Da Cruz Simões

Silva, Sofia

Silva-Abreu, Marcelle

Silveira, Nádya P. Da

Silvestri, Brigida

Simeone, Pasquale

Simoes, Sandra

Simon, Laurent

Singaraju, Aditya

Singh, Ajay Vikram

Singh, Ishwar

Singh, Mogie

Sinha, Abhijeet

Sinko, Patrick

Siracusa, Rosalba

Sitar, Daniel S.

Sivalingam, Periyasamy

Skalko-Basnet, Natasa

Skerra, Arne

Šklubalová, Zdeňka

Skotnicki, Marcin

Skouras, Athanasios

Slimano, Florian

Sloan, Alastair J.

Smaldone, Gerald C.

Smani, Younes

Smarandache, Adriana

Śmiga-Matuszowicz, Monika

Smith Callahan, Laura A.

Smit-Mcbride, Zeljka

Snežana, Papović

Soares, Graca M.B.

Sobczak, Marcin

Soheila, Ali Akbari Ghavimi

Soica, Codruta

Solimando, Antonio Giovanni

Solomon, Melani

Solopov, Pavel

Solorio, Luis

Song, Chunhua

Song, Im Sook

Song, Jianxun

Song, Liujiang

Song, Lusheng

Song, Xinhua

Song, Ziyuan

Sorrenti, Milena

Sotiriou, Georgios 
Soto, Fernando

Sou, Keitaro

Soulairol, Ian

Sour, Angélique

Sovány, Tamás

Sovinco, Fabio

Spanakis, Marios

Spartalis, Eleftherios

Spetea, Mariana

Spingler, Bernhard

Spoerk, Martin

Squadrito, Mario Leonardo

Squassina, Alessio

Srcic, Stanko

Sreedharan, Sreejesh

Sreerama, Subramanya

Stacchiotti, Alessandra

Stamm, Manfred

Stanek, Agata

Stankov, Karmen

Starynowicz, Przemyslaw

Stavros, Malamataris

Stawny, Maciej

Steenekamp, Jan

Stefanucci, Azzurra

Steiner, Denise

Štěpánek, F.

Stepanenko, Aleksei A.

Stepensky, David

Stephan, Holger

Steup, Martin

Stieger, Bruno

Stobiecka, Magdalena

Stoddard, Shana

Stolp, Helen

Stratford, Robert E.

Strickley, Robert

Stringaro, Annarita

$\mathrm{Su}$, Changqing

Su, Chie-Shaan

$\mathrm{Su}, \mathrm{Yeu}$

$\mathrm{Su}$, Zheng-Yuan

Suarato, Giulia

Subhan, Md Abdus

Suda, Kenichi

Suga, Hirotaka

Sugano, Kiyohiko

Sugimoto, Hiroshi

Sugiyama, Hirokazu

Suh, Hyung Joo

Sui, Yongjun
Sun, Caijun

Sun, Conroy

Sun, Hao

Sun, Jiazhen

Sun, Junjiang

Sun, Leming

Sun, Zhixiong

Sundararajan, Raji

Suntornnond, Ratima

Sur, Subhayan

Suresh, Kuthuru

Sutariya, Vijaykumar B.

Suzuki, Jon B.

Suzuki, Toyofumi

Swindle, Michael

Syed, Asad

Sykłowska-Baranek, Katarzyna

Sytar, Oksana

Szczepanowicz, Krzysztof

Székely, Andrea

Szeleszczuk, Łukasz

Szewczyk, Nathaniel J.

Szlęk, Jakub

Szulc-Dąbrowska, Lidia

Szwajca, Anna

Szweda, Piotr

Szymańska, Emilia

Tacconelli, Stefania

Tachibanaki, Shuji

Tadić, Vanja

Tagad, Harichandra

Tagit, Oya

Taglietti, Angelo

Tai, Wanbo

Tajber, Lidia

Takacs-Novak, Krisztina

Takács-Novák, Krisztina

Takahara, Mari

Takatani-Nakase, Tomoka

Tallapaka, Shailendra B.

Tallarico, Marco

Tambuwala, Murtaza

Tamošiūnas, Algirdas Edvardas

Tampa, Mircea

Tampucci, Silvia

Tan, Songwen

Tan, $\mathrm{Xu}$

Tanaka, Takuji

Tanaka, Tomonari

Tanc, Muhammet

Tang, Jing 
Tang, Yuan

Taranto, Alex Gutterres

Tatischeff, Irène

Tatu, Alin Laurențiu

Taurin, Sebastien

Tavakoli, Javad

Tavares, Anthony

Taylor, Rebecca E.

Tekko, Ismaiel

Teleki, Alexandra

Terao, Junji

Thackaberry, Evan A.

Thaker, Youg Raj

Thakur, Basant Kumar

Thakur, Sachin

Theile, Dirk

Thommes, Markus

Tian, Bo

Tian, Jiangwei

Tian, Justin

Tien, An-Chi

Tiera, Marcio

Tikoo, Suresh K.

Timmins, Peter

Ting, Jeffrey M.

Tiwari, Roshan

Todorov, Svetoslav Dimitrov

Togami, Kohei

Tokars, Valerie L.

Tomatsu, Shunji

Tomić, Simonida

Tomlin, Stephen R.

Tomuta, Ioan

Tong, Zhenbo

Topuz, Fuat

Torrado, Juan J.

Torrado-Santiago, Santiago

Torre, Maria Luisa

Torres-Suárez, Ana-Isabel

Tosi, Giovanni

Touraud, Didier

Toutain, Pierre Louis

Tran, Fabien

Tran, Phuong

Tran, TTD

Trandafirescu, Cristina-Maria

Trapani, Ivana

Tricarico, Domenico

Tripodo, Giuseppe

Trivedi, Vivek

Troutman, Jerry M.
Trucillo, Paolo

Trujillo De Santiago, Grissel

Trujillo-Cayado, Luis

Truong, Nghia P.

Truong, Vi-Khanh

Tsapis, Nicolas

Tsiourvas, Dimitrios

Tsiourvas, Dimitris

Tsirigotis-Maniecka, Marta

Tsukamoto, Tetsuo

Tsuneoka, Makoto

$\mathrm{Tu}$, Ting-Yuan

Tupally, Karnaker R.

Turcan, Sevin

Tyliszczak, Bożena

Tyurin-Kuzmin, Pyotr

Uchida, Satoshi

Ueng, Yune-Fang

Uhrikova, Daniela

Ulbert, Sebastian

Umerska, Anita

Ungaro, Francesca

Ungurianu, Anca

Upadhya, Manoj Atmaramji

Uppulury, Karthik

Urbančič, Iztok

Urbanczyk-Lipkowska, Zofia

Urbanelli, Lorena

Uritu, Cristina Mariana

Urschitz, Johann

Urso, Elena

Urtti, Arto

Uthaman, Saji

Uzbekov, Rustem E.

Vaidya, Amita

Vaidya, Bhuvaneshwar

Vaidyanathan, Ganesan

Vaidyanathan, Sriram

Valable, Samuel

Valente, Artur J. M.

Valsami, Georgia

Vamanu, Emanuel

Van Den Mooter, Guy

Van Riet-Nales, Diana A.

Vanaki, Shayan

Vance, David

Vandamme, Thierry

Vangala, Anil

Vangala, Venu

Vanic, Zeljka

Vanić, Zeljka 
Vaquero, Javier

Váradi, Csaba

Váradi, Judit

Varga, Zoltan

Varna, Mariana

Varum, Felipe

Vashist, Arti

Vasic, Vesna

Vasiliu, Silvia

Vazquez, Esther

Vázquez-López, Ezequiel M.

Vedagopuram, Sreekanth

Vée, Marc Le

Veeravalli, Vijayabhaskar

Vel, Bharath Kumar

Velasco, Rebeca Hernandez

Velikova, Tsvetelina

Velikyan, Irina

Vemulapalli, Vidyasiri

Venkatadri, Rajkumar

Venkatesan, Jayachandran

Ventura, Cinzia Anna

Veréb, Zoltán János

Vergara, Daniele

Vergnes, Bruno

Verron, Elise

Veszelka, Szilvia

Vetvicka, Vaclav

Vida, Yolanda

Vidal, Fernando

Víglaský, Viktor

Vijayakumar, Sekar

Vilella, Antonietta

Villarreal-Gómez, Luis Jesús

Vilsinski, Bruno Henrique

Vinarov, Zahari

Vine, Kara L.

Vingolo, Enzo Maria

Vitale, Fabrizio

Vitorino, Carla

Vivas Mejia, Pablo

Vivero-Escoto, Juan

Vives, Joaquim

Vlahovic, Tracey

Vlaia, Lavinia

Vlase, Gabriela

Vlase, Titus

Vogelbaum, Michael A.

Voliani, Valerio

Vora, Lalitkumar

Vorobyeva, Anzhelika
Voshavar, Chandrashekhar

Vostinaru, Oliviu

Vrečer, Franc

Vuddanda, Parameswara Rao

Vukmirovic, Sasa

Vulto, Arnold G.

Vuorimaa-Laukkanen, Elina

Waczulíková, Iveta

Wagner, Karl G.

Walter, Fruzsina R.

Wang, Chenguang

Wang, Fuke

Wang, Fuyi

Wang, Lei

Wang, Lingle

Wang, Pengcheng

Wang, Qun

Wang, Shi-Bin

Wang, Tao

Wang, Wei

Wang, Wenxin

Wang, Xiaodong

Wang, Zhenping

Wang, Zhijun

Wang, Zhisong

Wang, Zongjie

Warnken, Zachary

Warzecha, Zygmunt

Watts, Alan

Wei, Gang

Wei, Hua

Wei, Kai-Che

Weighardt, Heike

Weiss, Clemens K.

Weiss, Johanna

Weitschies, Werner

Weng, Yung-Jin

Wertz, Philip W.

Wesołowski, Marek

Westergaard, Niels

Wibowo, David

Wiener, Zoltán

Wietrzyk, Joanna

Wiglusz, Rafał Jakub

Wildemann, Britt

Williams, Gareth

Williams, Noelle S.

Wilner, Samantha E.

Winnicka, Katarzyna

Witkowska Nery, Emilia

Wojciechowski, Kamil 
Wölk, Christian

Wołowiec, Stanisław

Wong, Boon Seng

Wong, Chin-Chean

Wong, Dennis CC

Woźniak-Budych, Marta

Wright, David

$\mathrm{Wu}$, Huan

$\mathrm{Wu}$, Jun

$\mathrm{Wu}, \mathrm{Wei}$

$\mathrm{Wu}, \mathrm{Yu}$-Tse

$\mathrm{Wu}$, Zhangxiong

Wurm, Florian

Wuttke, Stefan

Xi, Guifa

Xia, Tian

Xiao, Chunsheng

Xing, Gengmei

Xiong, Jia

Xiong, Zhaohui

$\mathrm{Xu}$, Cancan

$\mathrm{Xu}, \mathrm{Lu}$

Xu, Pengfei

$\mathrm{Xu}$, Rong

$\mathrm{Xu}, \mathrm{Tao}$

$\mathrm{Xu}$, Xiao-Ding

$\mathrm{Xu}$, Yan-Ming

Xue, Jiajia

Xue, Ming

Yadavalli, Tejabhiram

YAKAVETS, Ilya

Yallapu, Murali

Yallapu, Murali Mohan

Yan, Dayun

Yan, Lifeng

Yan, Xuehai

Yaneva, Zvezdelina L.

Yang, Chih-Tsung

Yang, Chul-Su

Yang, Chunhua

Yang, Fang

Yang, Fengqing

Yang, Hung-Wei

Yang, Jianmin

Yang, Kai-Chiang

Yang, Seung Yun

Yang, Xiaochun

Yang, Yannan

Yang, Yongkang

Yaoita, Yasunori

Yapp, Donald T.
Yaraki, Mohammad Tavakkoli

Yasuda, Takako

Yasunaga, Masahiro

Yazdi, Iman

Yazdimamaghani, Mostafa

Yen, Meng-Chi

Yeo, Syn

Yi, Tao

Yildirim, Adem

Yilmaz, Catalina Natalia Cheaburu

Yilmaz, Mehmet

Yin, Guowei

Yin, Jun

Yokota, Shin-ichi

Yokoyama, Shinji

Yonemochi, Etsuo

Yong, Kar Wey

Yongxiang, Luo

Yoo, Hyuk Sang

Yook, Simmyung

Yoon, Bo-Eun

Yoon, In Soo

Yoshida, Kentaro

Yoshii, Hidefumi

Yoshioka, Ken-ichi

Younes, Husam

Young, Howard A.

$\mathrm{Yu}$, Deng-Guang

$\mathrm{Yu}, \mathrm{Lu}$

$\mathrm{Yu}$, Weiling (Connie)

Yuan, Wei-En

Yuan, Zhihong

Yuan, Zhiqin

Yuba, Eiji

Yue, Pengfei

Yumura, Shigehiko

Yutani, Reiko

Yu-Wai-Man, Cynthia

Zabielska-Koczywąs, Katarzyna

Zaboronok, Alexander

Zaccheroni, Nelsi

Zahari, Vinarov

Zahid, Maliha

Zaid, Abdel Naser

Zaman, Farasat

Zambito, Ylenia

Zamostny, Petr

Zamparini, Fausto

Zapotoczny, Szczepan

Zarzuelo Castañeda, Aránzazu

Zarzycki, Pawel K. 
Zdarta, Agata

Zdarta, Jakub

Zelkó, Romána

Zeng, Bijun

Zeni, Olga

Zhang, Dianbao

Zhang, Hongbo

Zhang, Huijie

Zhang, Jinjin

Zhang, Liqiang

Zhang, Rujing

Zhang, Tianyu

Zhang, Weizhong

Zhang, Xiaoyu

Zhang, Xingmin

Zhang, Xuan

Zhang, Xunli

Zhang, Yajie

Zhang, $\mathrm{Yi}$

Zhang, Yu

Zhang, Yue

Zhang, Yufeng

Zhang, Yumiao

Zhao, Chunxia

Zhao, Min

Zhao, Qinjian

Zhao, Xiuhua

Zhao, Yuanli

Zhao, Zhenjiang

Zheng, Xinting

Zhou, Dongfang
Zhou, Dongming

Zhou, Jichun

Zhou, Kun

Zhou, Qun

Zhou, Yubin

Zhou, Zhuxian

Zhu, Caihong

Zhu, Chunlei

Zhu, Hongyan

Zhu, Jingen

Zhu, Lan

Zhu, Limin

Zhu, Yishen

Ziaee, Ahmad

Zidek, Jan

Ziegler-Borowska, Marta

Ziemssen, Focke

Zingg, Jean-Marc

Zmudzki, Pawel

Zobi, Fabio

Żołek, Teresa

Zorov, Dmitry B

$\mathrm{Zu}$, Youli

Zuber, Guy

Zuccari, Guendalina

Žugić, Ana

Zúñiga, Leandro

Zurth, Christian

Zvonar, Alenka

Zydowsky, Thomas M

Zyuzin, Mikhail V. 